\section{CPC-100 PHARMACEUTICAL CARE IN PATIENTS DIAGNOSED WITH MULTIPLE MYELOMA TREATED WITH LENALIDOMIDE}

doi:10.1136/ejhpharm-2013-000276.557

'P Carmona Oyaga, 2M Iglesias Gaspar, 'G Lizeaga Cundin, II Fernandez Gonzalez, 'O Valbuena Pascual, 'P Pascual Gonzalez, ' 'J Barral Juez, 'M Umerez Igartua, 'A Asensio Bermejo, 'MP Bachiller Cacho. 'Donostia University Hospital, Pharmacy Service, San Sebastián, Spain; ²Donostia University Hospital, Epidemiology Service, San Sebastián, Spain

Background Multiple myeloma (MM) is a malignant monoclonal gammapathy that occurs mainly in patients over 65 years. Lenalidomide is indicated in combination with dexamethasone for the treatment of $\mathrm{MM}$ in patients who have received at least one prior treatment regimen.

All this makes it likely the patient will require Pharmaceutical Care (PC). PC consists of collaboration with other health professionals and with the patient to design a safe and effective treatment plan, as well as to identify Drug Related Problems (DRPs) and to resolve and prevent negative outcomes associated with medication (RNMs). Purpose To evaluate the impact of pharmaceutical intervention in patients diagnosed with MM treated with lenalidomide in a pharmacists-led haematological consultation within the Pharmacy Service.

Materials and Methods Quasi-experimental study of 4 months duration on patients diagnosed with $\mathrm{MM}$ treated with lenalidomide. Clinical practise follow-up procedures used the Dader method adapted to the study situation. Data were obtained from interviews with patients, electronic medical records and Outpatient Service Pharmacy records.

Results During this period, 29 patients were diagnosed with MM and treated with lenalidomide, 21 joined the study (4 didn't gave consent and 2 weren't able to visit the pharmacy), 11 women and 10 men. Average age: 70.3 years (52-89). During study a total of 17 DRPs were detected: 4 related to the indication, 1 to the effectiveness and 8 to the safety, and a total of 35 RNMs: 4 related to the need, 5 to the effectiveness and 26 to the safety. Of these $35,45.7 \%$ could have been avoided. A total of 25 pharmaceutical interventions were made: 10 related to the amount of drug, 9 to the pharmacological strategy and 6 to patient education.

Conclusions A variety of goals were achieved through pharmaceutical interventions: medicines reconciliation, resolution of health problems by detecting RNMs and avoidance of RNMs by detecting DRPs.

No conflict of interest.

\section{CPC-101 PHARMACEUTICAL INTERVENTION IN THE PATIENT RECORD: TOWARDS HARMONISATION OF OUR PRACTISE}

doi:10.1136/ejhpharm-2013-000276.558

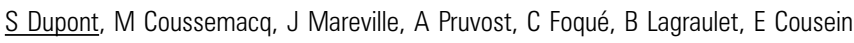
Centre Hospitalier de Valenciennes, Pharmacie, Valenciennes, France

Background In our hospital, patient records and all medical prescriptions are computerised in 11 departments. These prescriptions are analysed daily by a pharmacist. Each pharmaceutical intervention (PI) is recorded in the patient record and can be accepted or rejected by physicians. PIs are marked critical, medium or low by the pharmacist. We set up a weekly PI meeting with all pharmacists in June 2012. Purpose To standardise, analyse and promote our interventions. Materials and Methods For each PI, the pharmacy student fills in an Excel table with medical ward, drug, problem identified, type of intervention, pharmacist rating and clinical impact of the intervention. During the meeting, all PIs marked critical or that had a physician comment, discrepancy on out of formulary discharge proposal, or any IPs considered relevant by the pharmacist were considered and discussed.

Results Analysis of medical prescriptions generated 1,483 PIs over 3.5 months. The most frequent rating was 'low' (70\%). There were
18\% 'medium' and 3\% 'critical' interventions. The main pharmaceutical problem was out of the formulary discharge proposal which represented $54 \%$ of PIs (796/1483). Dosage adaptation was recommended in $12 \%$ of cases; $9 \%$ of PIs were for stopping the treatment and other interventions were about the choice of route of administration, adding a treatment, therapeutic monitoring and optimization of administration. In total, $58 \%$ of PIs were accepted, the physician was not informed of $23 \%$ and $19 \%$ were not accepted; but $11 \%$ of the PIs accepted were not implemented.

135 PIs were discussed in pharmaceutical meetings. Among the subjects that arose, 3 were particularly highlighted: re-evaluation of renal failure and metformin, interaction between beta blockers and flecainide and recommendations on allergies. We have studied out of the formulary discharge proposal discrepancies about cardiology medicines (angiotensin converting enzyme inhibitors and angiotensin receptor antagonists).

Conclusions Feedback on PIs is a key element to improve their relevance. Finally, a weekly pharmaceutical meeting can highlight recurrent prescription problems in order to propose and implement corrective measures. It is moreover a working base for our hospital to improve the quality of medical care.

No conflict of interest.

\section{CPC-102 PHARMACIST-DRIVEN INTERVENTIONS IMPROVE THROMBOPROPHYLAXIS IN ACUTELY ILL MEDICAL PATIENTS OVER TIME - RESULTS AFTER SIX MONTHS}

doi:10.1136/ejhpharm-2013-000276.559

'A Vervacke, 'S Lorent, ${ }^{2} \mathrm{M}$ Mrabtifi, ${ }^{2} \mathrm{~S}$ Motte. 'Erasme University Hospital, Pharmacy, Bruxelles, Belgium; ' $E$ rasme University Hospital, Thrombosis Unit, Bruxelles, Belgium

Background Forty to $50 \%$ of hospitalised patients with an acute medical illness have risk factors for venous thromboembolism (VTE) and it has been shown that thromboprophylaxis reduces the incidence of VTE events in these patients [1]. However, a large multinational survey, the ENDORSE study, showed that only $37 \%$ of medical patients with VTE risk factors currently received thromboprophylaxis [2].

Purpose To evaluate the impact over time of pharmacist-driven interventions aiming at increasing the appropriate use of thromboprophylaxis in acutely ill medical patients hospitalised in an urban academic tertiary care hospital.

Materials and Methods First, medical and nurse reports of hospitalised medical patients were reviewed to evaluate the proportion of patients who were on prophylaxis according to clinical practise guidelines. Second, interventions were conducted and included unit-specific physician and nurse education, dissemination of educational tools summarising VTE prophylaxis guidelines, and reminders. Third, the effect of the interventions on the proportion of patients receiving appropriate thromboprophylaxis was evaluated after three and six months.

Results The baseline evaluation showed that $36 \%(26 / 72)$ of the patients at risk of VTE received appropriate thromboprophylaxis. Three and six months after the interventions, 68\% (55/81), and $72 \%(58 / 81)$ of the patients at risk of VTE received appropriate thromboprophylaxis.

Of the patients not at risk of VTE, 15\% (21/141), 8\% (24/290), and $8 \%(27 / 330)$ respectively at baseline evaluation, three and six months after the interventions, received thromboprophylaxis.

Conclusions Pharmacist-driven interventions improved the proportion of acutely ill medical patients receiving appropriate thromboprophylaxis and the benefit of the interventions was maintained after six months.

\section{References}

1. Alikhan Ret al, Blood Coagul Fibrinolysis, 2003;14:341-6.

2. Mahe I., Drugs \& Aging, 2007, 24(1): 63-71

No conflict of interest. 